\title{
Identificación Social y/o Percepción de Similitud: Aproximación a los Efectos Diferenciales sobre el Esencialismo ${ }^{1}$
}

\author{
Katherine Saldivia Ojeda \\ Débora Mansilla Cofré \\ Claudia Andrea Estrada Goic ${ }^{2}$ \\ Universidad de Magallanes
}

\begin{abstract}
RESUMEN - La presente investigación trata de la relación causal de dos procesos asociados al esencialismo (creer en la existencia de una esencia común a todos los miembros de un grupo): la identificación social y la similitud. Su objetivo fue determinar el rol de ambos procesos sobre el esencialismo psicológico. Mediante un estudio transversal correlacional, 200 personas (muestreo no aleatorio) fueron medidas en ambas variables y en esencialismo (endo y exogrupal). Los resultados indican efectos diferenciales según el tipo de esencialismo (endo o exogrupal). El endogrupal es explicado por la identificación grupal (autocategorización y compromiso grupal). El exogrupal, depende de la identificación (autocategorización) y la similitud (homogeneidad). Se discuten estos resultados en torno a sus consecuencias para las relaciones intergrupales.
\end{abstract}

Palabras-clave: psicología social, esencialismo, identidad social

\section{Social identification and / or perception of similarity: An approach to differential effects on ingroup and outgroup essentialism}

\begin{abstract}
The present study deals with the causal relation among two processes associated to essentialism (believing in the existence of a common essence for the members of a group): social identification and similarity. By means of a crosscorrelational study, 200 participants (non-random sampling) were measured on both variables and on essentialism (ingroup/ outgroup). The results indicate that the effects of both variables are not similar according to whether they belong to ingroup or outgroup essentialism. Ingroup essentialism is mainly explained by identification with the ingroup, and, especially for selfcategorization and group commitment. The outgroup essentialism, meanwhile, is explained by self-categorization (identification) and the perception of intragroup homogeneity (similarity). These results are discussed in relation to their implications for intergroup relations.
\end{abstract}

Keywords: social psychology, essentialism, social identity

El esencialismo psicológico puede definirse como la tendencia a creer que la pertenencia a un grupo o categoría social es el producto de la existencia de una esencia común a todos sus miembros (Medin, 1989). Es un constructo que se inserta dentro de las llamadas "Teorías Implícitas", que han sido definidas como un conjunto de creencias relativamente estables que, contenidas en un individuo, pueden ser compartidas por un grupo, actuando implícitamente en sus actitudes y, consecuentemente, en su comportamiento (Corneille \& Leyens, 1994). Se le clasifica como tal, dentro de la subcategoría de las teorías implícitas sobre el origen de los grupos. Rothbart y Taylor (1992) postularon que el esencialismo es el resultado de un "error", que consiste en comprender a las categorías sociales como si estas estuviesen dotadas de una "realidad" similar a las categorías naturales, que diferencian, por ejemplo, a las plantas de los animales. Las personas "olvidamos" que los grupos son el resultado de decisiones y contextos sociales, y comenzamos a verlas como

1 Financiado por Grant FONDECYT 1140947

2 Endereço para correspondência: Centro de Estudios en Psicología Social (CEPS), Escuela de Psicología, Facultad de Humanidades y Ciencias Sociales, Avda. Bulnes 01855, Punta Arenas, Chile. E-mail: claudia.estrada@umag.cl dotadas de esencias naturales que explican su concurrencia. Aunque fue Sherif (1948) quien tempranamente observó que ciertas personas tienden a describir eventos sociales en términos de la presencia de "esencias", fue Allport (1954) quien utilizó el término esencialismo por primera vez en su célebre libro sobre el prejuicio. Postuló que el que los observadores percibieran al grupo social como dotado de componentes comunes a todo sus miembros, facilitaba la ocurrencia del prejuicio. Según Allport (1954), este fenómeno respondía a la necesidad cognitiva individual de realizar el menor esfuerzo posible al momento de juzgar a los otros. Es así como el esencialismo fue definido como una creencia que genera consecuencias negativas en las relaciones sociales pero a su vez crea positivas para el funcionamiento individual. En términos generales, y aunque se han descrito una serie de características que describen el síndrome esencialista (Yzerbyt \& Schadron, 1996), existe acuerdo en que las categorías asociadas a cuestiones biológicas, suelen esencializarse con mayor facilidad. El determinismo genético ha sido señalado como un elemento fundamental en la creencia de que existe una esencia común, escrita en "la sangre", anclada en factores biológicos (Pereira, Estramiana, Vasconcelos, \& Alves, 2010; Zagefka, Nigbur, Gonzalez, \& 
Tip, 2013). Martin y Parker (1995) evidenciaron que cuando un individuo privilegia la dimensión biológica al momento de explicar las diferencias entre sexos y raza, presenta también intolerancia a la ambigüedad y resistencia al cambio. Este fenómeno sucedería debido a que se mantiene la creencia de que existen características estables e inmutables que explican la existencia de los grupos más que en la presencia de otros factores que pueden ser más dinámicos, como los culturales y sociales. En términos generales, las consecuencias observadas del esencialismo en el plano intergrupal son numerosas e incluyen: el aumento del prejuicio respecto de miembros minoritarios (Zagefka et al., 2013), o hacia un exogrupo (Estrada, Oyarzún, \& Yzerbyt, 2007), la infrahumanización (Leyens et al., 2000; Leyens et al., 2001), la legitimidad de los estereotipos (Pereira, Alvaro, Oliveira, \& Dantas, 2011; Yzerbyt \& Rocher, 2002; Yzerbyt, Rocher, \& Schadron, 1997), y el sesgo endogrupal (Estrada, 2009, Estrada et al., 2007). Asimismo existen evidencias respecto de su impacto en procesos intragrupales tales como: el aumento de la proyección social, percepción de prototipicalidad, mayor percepción de similitud endogrupal, etc. (Calquín \& Estrada, 2011). Actualmente las investigaciones sobre esta temática se centran en el dinamismo del esencialismo en diferentes contextos relacionales y políticos (Morton \& Postmes, 2009; Pehrson, Brown, \& Zagefka, 2009). En este sentido, los estudios exploran su carácter motivacional y las posibles consecuencias positivas que tiene para procesos asociados a la identidad social. En este contexto, los procesos clásicos que han sido utilizados para observar el efecto de variables causales sobre las creencias esencialistas han considerado dos variables: la identificación con el grupo y la percepción de similitud con el mismo. Las características de los grupos "esencializados" son, según Yzerbyt y Schadron (1996): 1) Estatus ontológico específico: el grupo es percibido como una entidad, es decir, tiende a ser visto como homogéneo; 2) Inalterabilidad de la pertenencia categorial: cuando una persona pertenece a un grupo específico no puede pertenecer a otro; 3) Carácter inductivo: cuando conocemos que una persona pertenece a un grupo particular creemos tener más información o saber más ampliamente sobre él; 4) Fuertes conexiones entre los atributos de una categoría: los atributos se relacionan y organizan de forma coherente, de esta forma son interpretados como un todo unificado. Las personas esencializadas asumen la existencia de una relación de causa y efecto entre la posesión de la esencia y el conjunto de características que ostenta el grupo social; 5) Exclusión de otras formas de conocimiento y aprensión del individuo: la persona se aferra a sus creencias al momento de vincularse $o$ establecer un tipo de contacto con un grupo en particular. Además se le ha vinculado con nociones tales como el concepto de "entitatividad" (Campbell, 1958) en el que la concepción de pertenecer a un grupo con existencia real, se construye a partir de la proximidad de sus miembros, la similitud de los elementos de un grupo y el destino común. Desde esta perspectiva, la vinculación entre esencialismo y similitud ha sido ampliamente establecida (Demoulin, Leyens, \& Yzerbyt, 2006; Estrada, Yzerbyt, \& Seron, 2004; Yzerbyt \& Demoulin, 2010). Algunas investigaciones señalan que la entitatividad u otros conceptos asociados como la "naturalización" (tendencia a comprender las categorías sociales como naturales) dependen del tipo de grupo en el que se esté evaluando la adhesión a creencias esencialistas (Demoulin et al., 2006). La teoría de la similitud-atracción postula que la sola semejanza real o percibida entre los individuos facilita la atracción y, en consecuencia, la disposición a vincularse con el grupo (Byrne, 1971). Es así que el esencialismo, al tratarse de una creencia en la que se define que la existencia de una "esencia" se exterioriza mediante similitudes visibles, conducirá a pesar que las similitudes de superficie responden a la acción de elementos profundos que las determinan. Lo anterior condiciona a la persona a una representación mental de sí mismo y del grupo como "similares", llevando al primero a posicionarse como prototípico del grupo (Calquín \& Estrada, 2011). El proceso de similitud puede ser entendido como compuesto en cuatro subclases: La primera de ellas es el "autoestereotipo" que se refiere a la percepción que tiene el sujeto sobre las características que debiese tener un representante típico de su grupo (Alarcón \& Estrada, 2011; Kamans, Gordijn, Oldenhuis, \& Otten, 2009). El segundo y tercer tipo de similitud son los postulados por Codol (1979), quien define la existencia de dos tipos de similitud en la relación entre el individuo y su grupo. Por una parte encontramos aquella referida al "individuo-grupo", que tiene relación a cómo el sujeto percibe que él como individuo posee características similares a las del grupo al que pertenece ("yo me parezco a ellos"). Por otra parte, aquella referida a la similitud "grupo-individuo" pone acento en el parecido que el grupo tiene con el individuo ("ellos se parecen a mí"). Finalmente, se encuentra la similitud como homogeneidad intragrupo, definida como la percepción de que las características de los miembros de un grupo son semejantes entre sí (Montalbán \& Gómez, 1995).

La identificación, por su parte, ha sido considerado un proceso central que da cuenta de cómo pertenecer a una determinada categoría social impacta en la identidad del un individuo. Es cuando dicha persona se reconoce como miembro de un grupo, le otorga valor y se siente comprometido con él, es que sabemos que su membrecía se ha constituido en una parte significativa de quién es.

La Teoría de la Identidad Social (TIS), fue originalmente postulada por los trabajos de Henry Tajfel en las década de los cincuenta, en el área de la percepción categorial (Tajfel, 1957). Plantea la existencia de tres procesos centrales en la construcción de la identidad social entendida como aquella parte de lo que se es, que está constituida por nuestra pertenencia a grupos y categorías sociales. El primero es el de categorización social: organizamos y simplificamos nuestro entorno social para entenderlo, para darle sentido, es decir, lo ordenamos en categorías, ya sea acentuando similitudes entre los miembros de un grupo o bien enfatizando las diferencias entre los miembros de otros grupos. El segundo es el proceso de comparación social: consiste en poner en perspectiva las características tanto propias como las de otros grupos de forma a establecer diferenciaciones en las que unos resultan mejores (más valiosos) que otros. Naturalmente emerge la necesidad de comparar al propio grupo con otros grupos o categorías en las que se saldrá favorecido, lo que ha sido denominado distintividad social positiva. Finalmente, encontramos el proceso de identificación social, 
que consiste en reconocerse como miembro de un grupo e identificarlo como parte de lo que somos. El éxito de estos procesos se traduce en el logro de una identidad social positiva. El autoconcepto se ve fortalecido por la pertenencia a grupos que son valorados socialmente. En otros términos, la identidad social tendería a maximizar la autoestima mediante la identificación con todos aquellos grupos sociales específicos a los que pertenece el individuo e intentando que sean, a comparación de los otros grupos, valorados de forma positiva. Sin embargo, que la valoración sea positiva o negativa, dependerá de la evaluación que realice el individuo de su grupo en comparación con otros grupos, por lo que es al mismo tiempo, el resultado de la percepción individual y del contexto social. Desarrollos posteriores de Turner (1982), ampliaron las ideas de Tajfel en la dirección de plantear que la identificación social puede tener como consecuencia el comportamiento grupal. Según sus postulados, los individuos pueden situarse en diferentes niveles de identificación con el grupo. Cuando la identificación es mínima, los individuos se ven a sí mismos como tales, en un proceso que denominó individuación. En el otro extremo, cuando la identificación es máxima, el individuo se percibe a sí mismo como miembro de un grupo, lo que ha sido denominado despersonalización. Las consecuencias de la identificación social son la tendencia a ser más cooperativo con los miembros de su endogrupo, la necesidad de estar de acuerdo con las opiniones de su grupo, y la orientación a percibir los mensajes de su grupo como de mejor calidad que los del exogrupo. Hinkle, Taylor, Fox-Cardamone y Crook (1989) desarrollaron una escala para medir tres aspectos de la identificación grupal: cognitivo (saber que soy miembro de un grupo social), evaluativo (tiene relación con la connotación positiva o negativa que le entrego al grupo) y emocional (sentirse involucrado emocionalmente con el grupo). El modelo de distintividad óptima de Brewer (1991) plantea que las personas, al establecer su identidad, deben satisfacer simultáneamente las necesidades de integración (afiliación) y diferenciación. La identificación comportaría, en sincronía con las ideas plantadas por Tajfel (1984), tres elementos: a) autocategorización: La consciencia cognitiva de la pertenencia al grupo (cognitivo), b) compromiso afectivo: El grado en que uno se siente emocionalmente implicado con él (emocional), y c) autoestima grupal: El valor que se atribuye a la pertenencia a dicho grupo (evaluativo). Sin bien estos aspectos parecen estar fuertemente relacionados, esto no implica que siempre estén unidos, ni que se presenten con la misma fuerza (Ellemers, Kortekaas, \& Oawekerk, 1999). En otros términos, la identificación social comporta elementos perceptivos, afectivos y emocionales que la conforman como un proceso actitudinal complejo.

Las creencias esencialistas podrían tener un rol activo en la construcción de la identidad social, participando de los procesos que la constituyen (categorización social, comparación social e identificación social). La categorización social puede estar vinculada con las creencias esencialistas ya que determinan, como hemos establecido anteriormente, la percepción de las características que establecen la existencia de un grupo u otro. Estas creencias también afectan el proceso de comparación social puesto que definen la existencia de una esencia única para cada grupo donde la propia tiende a ser superior en alguna dimensión relevante. La comparación con bases esencialistas, determina tanto qué características serán relevantes, como la inalterabilidad de la pertenencia a cada grupo. La identificación, que en el caso esencialista tiene bases perceptivas (la esencia común), también comporta otros elementos en torno a la idea de que las fronteras del grupo son impermeables por lo que la pertenencia al mismo no es una decisión, sino una consecuencia de la esencia. De esta manera, el esencialismo, como creencia sobre el origen de los grupos, entrega estabilidad a la identidad social, pues ésta se establece a partir de elementos invariables que se encuentran en la "esencia". Cuando un grupo cree fuertemente en la existencia de una "esencia" común en todos sus miembros, su identidad ha sido "fundada" y será mantenida por esta creencia. A su vez, cuando el proceso de identificación social "sentirse parte de un grupo", es compartido como una creencia inicial, se favorecerá el desarrollo y mantención de la identidad social. Posteriormente la creencia podrá transformarse en esencialista al definir que "somos parte de un grupo porque tenemos una esencia en común". Wagner, Holtz y Kashima (2009) postulan que el esencialismo actúa como una herramienta representativa que es utilizada para crear la identidad de grupos y que es funcional cuando se busca aumentar la visión de que el mismo es una entidad real. De acuerdo a las características que hemos descrito, tanto la identificación social como el esencialismo poseen componentes cognitivos que vinculan lo individual con lo grupal, lo que explicaría su tendencia a correlacionar.

En el análisis y entendimiento del proceso esencialista son de primordial importancia ambos modelos: percepción de similitud, "que tan homogéneos y/o similares podemos ser" e identificación "sentirse parte de un grupo". En la actualidad ambos mediadores son utilizados en la investigación sobre el desarrollo de creencias esencialistas, no obstante no se sabe con exactitud si ambas explican por sí solas este fenómeno o actúan en conjunto. Algunos autores dicen que es percepción de similitud la que actúa de forma positiva en este fenómeno (e.g. Yzerbyt, Judd, \& Corneille, 2003), no obstante otros mencionan la identificación como mediador relevante para el proceso esencialista (Bastian \& Haslam, 2008). Ante esto surge la interrogante de cómo estas variables intervienen en el esencialismo, es decir, si son ¿La identificación y la Similitud son variables que explican el esencialismo psicológico?

\section{Método}

\section{Muestra}

La muestra no aleatoria accidental estuvo compuesta por 200 personas, de las cuales 189 se caracterizaron a sí mismas como "magallánicas" (Pertenecer a la región geográfica "Magallanes" nombre que recibe el territorio geopolítico de la Patagonia austral chilena), criterio de inclusión para el estudio. Un $56 \%$ de los participantes fue de género femenino mientras que el $44 \%$ restante fue de género masculino. Las edades de los encuestados varían entre los 18 y los 59 años de edad, siendo los más representados aquellos participantes que tienen 23 años (Mo). 


\section{Diseño}

Se utilizó un diseño no experimental, transversal, correlacional.

\section{Instrumentos}

Escala de identificación (adaptación del instrumento original de Ellemers, Kortekaas, \& Oawekerk, 1999): Compuesto de nueve ítems de la escala original traducidas al español y sometidas a una aplicación piloto para verificar su calidad psicométrica en esta versión. La escala consta de tres categorías que contienen tres ítems cada una, evaluadas sobre escalas Likert de cinco puntos (desde 1: "para nada de acuerdo" hasta 5: "totalmente de acuerdo"). Cada una de las categorías evalúa diferentes aspectos de la identidad social, "autoestima grupal" (ítems del 1 al 3), "autocategorización" (ítems del 4 al 6) y "compromiso con el grupo" (ítems del 7 al 9). Su confiabilidad para esta muestra fue de $0,90(\alpha)$.

Escala de similitud: Escala construida para la presente investigación con el objetivo de determinar la percepción de similitud entre el participante y su endogrupo. Esta escala contiene cuatro ítems que miden autoestereotipo, similitud individuo-grupo, similitud grupo-individuo y homogeneidad intragrupo. Dichos ítems son evaluados sobre escalas tipo Likert de cinco puntos (siendo 1: "Para nada de acuerdo" y 5 "Totalmente de acuerdo"). Su confiabilidad para esta muestra fue de $0,86(\alpha)$.

Escala de esencialismo (Estrada, 2003): escala construida para determinar la presencia de creencias esencialistas a la base de una categoría social. Esta escala contiene 11 ítems evaluados sobre escalas tipo Likert de seis puntos (desde 1: "para nada de acuerdo" hasta 6: "totalmente de acuerdo"). La escala posee una versión endogrupal (Magallánicos) y otra exogrupal (Coyhaiquinos, exogrupo de la Patagonia). Ambas versiones de la escala fueron respondidas por la misma muestra. La confiabilidad para esta muestra fue de 0,94 y 0,93 respectivamente.

\section{Procedimiento}

La aplicación de los instrumentos se realizó de forma individual (autoaplicada) y voluntaria dentro de un laboratorio en una sola sesión. Se les explicó el objetivo del estudio y se les entregó el consentimiento informado para que pudiesen ser firmados, posteriormente se entregaron uno a uno los cuestionarios, y una vez finalizado se les agradeció su participación.

\section{Resultados}

\section{Análisis Descriptivos}

El promedio general de identificación con el grupo alcanzó a un 4,2 (DE: 0,64), de un máximo de 5, superior a la media de investigaciones anteriores realizadas por el mismo equipo y con muestras similares, $M=3,9, t(199)=6,95, p=$
0,001 . Los tres factores de la escala, autoestima grupal $(M=$ $4,48 ; D E=0,60)$, autocategorización $(M=4,00 ; D E=0,84)$, y compromiso grupal $(M=4,20 ; D E=0,70)$, mostraron promedios significativamente sobre el punto neutro.

En el caso de la similitud el promedio general alcanzó a un $4,0(D E=0,84)$, de un máximo de 5 , superior a la media de investigaciones anterior $M=3, t(199)=10,00, p=0,001$. Tanto el factor autoestereotipo $(M=4,00 ; D E=1,09)$, como el de similitud individuo - grupo $(M=3,40 ; D E=1,00)$, el de similitud grupo - individuo $(M=4,00 ; D E=1,00)$ y el de homogeneidad intragrupo $(M=4,00 ; D E=0,94)$, resultaron estadísticamente superiores al punto neutro de la escala. La comparación entre las similitudes "individuo-grupo" y "grupo-individuo", indican que esta última presenta un promedio significativamente mayor, $t c_{(199)}=-8,4, p=0,001$.

El esencialismo endogrupal presentó un promedio de 5,00 (DE: 1,07), de un rango de respuesta de 1,0 a 6,0, superior a punto neutro de investigaciones anteriores (Estrada \& Avendaño, 2008), $M=4,2, t(199)=4,16, p=0,001$. Por su parte, en la variable de esencialismo exogrupal el promedio fue de 4,09 (DE: 1,03), de un rango de respuestas de 1,0 a 6,0, superior al punto neutro teórico de la escala, $M=3, t(199)=$ $15,0, p=0,001$. Al comparar los promedios de esencialismo endogrupal y exogrupal, ambos muestran una diferencia significativa que favorece al esencialismo endogrupal, $t c_{(199)}$ $=8,43, p=0,001$. En otros términos, las personas ven más a su propio grupo como dotado de características esencialistas que al exogrupo.

Tabla 1. Correlaciones entre las variables principales

\begin{tabular}{lcccc}
\hline & $\mathbf{1}$ & $\mathbf{2}$ & $\mathbf{3}$ & $\mathbf{4}$ \\
\hline 1.Identificación & - & $* * 0,69$ & $* * 0,64$ & $* * 0,49$ \\
2.Similitud & & - & $* * 0,51$ & $* * 0,37$ \\
3.Esencialismo Endogrupo & & & - & $* * 0,77$ \\
5.Esencialismo Exogrupo & & & & - \\
\hline
\end{tabular}

\section{Análisis Correlacionales}

Como es posible observar, las variables implicadas en el estudio se encuentran correlacionadas positivamente (ver tabla 2). El esencialismo endogrupal se relaciona positivamente tanto con identificación como con similitud. Lo mismo ocurre para el esencialismo exogrupal. En el caso del esencialismo endogrupal, la correlación observada con la variable identificación resultó ser mayor a la observada con la variable similitud $(Z=1,94 ; p=0,05)$. En el caso del esencialismo exogrupal, su relación con ambas variables resultó ser equivalente $(Z=1,47 ; p=0,14$, n.s.). Al comparar las relaciones observadas entre los dos tipos de esencialismo y la identificación, aquella asociación observada para ambas variables en el contexto endogrupal resultó mayor que la observada en el contexto exogrupal $(Z=2,20 ; p=0,03)$. Para el caso de la similitud y su relación con los dos tipos de esencialismo, se observan diferencias marginalmente significativas $(Z=1,73 ; p=0,08)$ 
Tabla 2. Correlaciones entre los tipos de similitud, tipos de identificación y esencialismo.

\begin{tabular}{|c|c|c|c|c|c|c|c|c|}
\hline Variables & 2 & 3 & 4 & 5 & 6 & 7 & 8 & 9 \\
\hline Esencialismo endogrupal & $* * 0,77$ & $* * 0,52$ & $* * 0,34$ & $* * 0,43$ & $* * 0,37$ & $* * 0,47$ & $* * 0,63$ & $* * 0,59$ \\
\hline Esencialismo exogrupal & - & $* * 0,38$ & $* * 0,25$ & $* * 0,32$ & $* * 0,28$ & $* * 0,35$ & $* * 0,50$ & $* * 0,43$ \\
\hline Similitud 1 & & - & $* * 0,62$ & $* * 0,69$ & $* * 0,49$ & $* * 0,55$ & $* * 0,64$ & $* * 0,57$ \\
\hline Similitud 2 & & & - & $* * 0,68$ & $* * 0,52$ & $* * 0,46$ & $* * 0,54$ & $* * 0,47$ \\
\hline Similitud 3 & & & & - & $* * 0,54$ & $* * 0,59$ & $* * 0,68$ & $* * 0,57$ \\
\hline Similitud 4 & & & & & - & $* * 0,37$ & $* * 0,36$ & $* * 0,32$ \\
\hline $\begin{array}{c}\text { Identificación } \\
\text { Autoestima Grupal }\end{array}$ & & & & & & - & $* * 0,69$ & $* * 0,68$ \\
\hline $\begin{array}{c}\text { Identificación Autocat. } \\
\text { Grupal }\end{array}$ & & & & & & & - & $* * 0,77$ \\
\hline $\begin{array}{c}\text { Identificación } \\
\text { Compromiso Grupal }\end{array}$ & & & & & & & & - \\
\hline
\end{tabular}

\section{Análisis de Regresión}

Se realizó una regresión lineal en que esencialismo endogrupal fue la variable dependiente, y similitud e identificación las independientes. Ambas variables explican el $42 \%$ de la varianza, $F_{(2,197)}=70,25, p=0,0001$. La percepción de similitud con el endogrupo, es decir, qué tanto los "miembros del grupo se me parecen", resultó marginalmente significativa $\beta=0,13, p=0,09,95 \%$ IC $[-0,03$, $0,40]$. La identificación, por su parte, resultó significativa para explicar el esencialismo $\beta=0,55, p=0,0001,95 \% I C$ $[0,67,1,17]$. El realizar el mismo análisis anterior incluyendo los factores de la identificación (Autoestima grupal, Autocategorización y Compromiso grupal) y de la similitud (autoestereotipo, similitud individuo-grupo, similitud grupo-individuo y homogeneidad intragrupo), se observa un impacto diferencial de cada uno de ellos. Significativas resultan para explicar el esencialismo endogrupal, los factores de la variable identificación: autocategorización $(\beta=0,44$, $p=0,0001,95 \%$ IC $[0,31,0,80])$, compromiso grupal $(\beta=$ $0,24, p=0,008,95 \%$ IC $[0,096,0,64])$, y para la variable similitud, autoestereotipo $(\beta=0,21, p=0,012,95 \%$ IC $[0,05$, $0,36])$, y homogeneidad intragrupo $(\beta=0,20, p=0,003,95 \%$ IC $[0,076,0,76])$. Todas ellas explican el $46 \%$ de la varianza del esencialismo endogrupal, $F_{(7,198)}=24,86, p=0,0001$.

Asimismo, se realizó un análisis de regresión lineal con el esencialismo exogrupo como variable dependiente, y similitud e identificación como variables independientes, explicando el $24 \%$ de la varianza, $F_{(2,199)}=31,22, p=0,0001$. Mientras que la percepción de similitud no resultó ser explicativa de este tipo de esencialismo $(\beta=0,07, p=0,43$, n.s., 95\% IC [- 0,12, 0,29]), la percepción de homogeneidad entre los miembros del exogrupo (que tanto se parecen entre sí), si explica el esencialismo exogrupal $(\beta=0,13, p=0,03$, $95 \%$ IC $[0,03,0,08])$. La identificación resultó explicativa, $\beta=$ $0,44, p=0,0001,95 \%$ IC $[-0,44,0,99]$. La regresión en la que las variables independientes son segregadas en sus factores: identificación (Autoestima grupal, Autocategorización y compromiso grupal) y similitud (autoestereotipo, similitud individuo-grupo, similitud grupo-individuo y homogeneidad intragrupo), se observa un impacto diferencial de cada uno de ellos. Explicativos resultan, en el caso de los factores de la variable identificación, únicamente la autocategorización $(\beta=0,47, p=0,0001,95 \%$ IC $[0,29,0,85])$, y para la variable similitud, la homogeneidad intragrupo $(\beta=0,15, p$ $=0,047,95 \%$ IC $[0,02,0,34])$. Todas ellas explican el $29 \%$ de la varianza del esencialismo exogrupal, $F_{(7,198)}=11,1, p$ $=0,0001$.

\section{Discusión y Conclusiones}

El principal objetivo de este estudio fue examinar el impacto de los procesos de percepción de similitud e identificación social sobre las creencias esencialistas. Para el caso del esencialismo endogrupal, ambas variables explican una parte importante de la varianza indicando que se trata de fenómenos importante implicados en el proceso de adhesión a las creencias sobre la existencia de la esencia en el propio grupo. La identificación muestra ser más importante que la similitud, en particular en el caso de la autocategorización y el compromiso grupal. La autocategorización hace referencia a la consciencia cognitiva de la pertenencia al propio grupo (Tajfel, 1957). El esencialismo endogrupal no puede darse sin que la persona se reconozca fuertemente como parte de su grupo, ya que su componente principal radica en compartir una esencia que predetermina su pertenencia al mismo (Yzerbyt \& Schadron, 1996). En este sentido, la autocategorización resulta indispensable para el surgimiento y la mantención de la creencia en la esencia del grupo ya que no puede darse sin que el individuo se reconozca como poseedor de los elementos profundos que determinan su membrecía al mismo. El compromiso grupal, por su parte, involucra un componente emocional que conecta al individuo con el propio grupo (Tajfel, 1957). El esencialismo ha sido consistentemente asociado a la presencia del sesgo endogrupal (Estrada et al., 2007). Siendo, la membrecía al grupo esencialista tanto importante como inevitable (inalterable), la búsqueda de positividad del mismo compromete particularmente la identidad del individuo. La conexión emocional con el propio grupo podría ser el resultado de la situación recientemente expuesta. La autoestima grupal, como componente de la identificación, no mostró ser explicativa del esencialismo endogrupal. 
Cabe destacar que ésta hace referencia al valor que se le atribuye a la pertenencia a dicho grupo. Un grupo esencialista se caracteriza por fronteras impermeables e inalterables (Yzerbyt \& Schadron, 1996), por lo tanto las personas que pertenecen a él se ven a sí mismo como individuos prototípicos, es decir, que reúnen las características centrales de un miembro de dicho grupo (Calquín \& Estrada, 2011). En este sentido, la valoración a la pertenencia grupal resultaría innecesaria puesto que sería una consecuencia inevitable de poseer la esencia, y no corresponde a una elección o al desarrollo de algún mérito o característica particular que le ha hecho merecedor a esa pertenencia. En el caso de la similitud, sólo resultaron significativas al momento de explicar el esencialismo endogrupal, el autoestereotipo y la homogeneidad intragrupal. El autoestereotipo, que hace referencia a que tan típico o prototípico se siente una persona respecto a su grupo de pertenencia, ha sido una medida que ha mostrado conexiones con el esencialismo en estudios reportados previamente (Estrada, 2003). No se trata únicamente de la visión de ser similar al grupo o el grupo similar a sí mismo, sino de verse "dotado" de las características centrales del mismo, es decir, ser poseedor de la "esencia". Lo mismo ocurre, en menor grado con la homogeneidad intragrupo. El esencialismo se define como poseedor de un "estatus ontológico específico", en el que el grupo tiende a ser visto como homogéneo (Yzerbyt \& Schadron, 1996). En este sentido, las personas tienden a aceptar el estereotipo de sus respectivas categorías y al mismo tiempo diferenciarse (Brewer, 1991). Estudios previos muestran que las personas toleran mejor que el grupo se les parezca a ellos que el que ellos se parezcan a su grupo (Codol, 1979). Nuestros resultados muestran el fenómeno contrario ya que el promedio de acuerdo con la expresión "me parezco al grupo", resultó mayor. Es posible que este resultado tenga relación justamente con altos promedios de esencialismo observados consistentemente en población magallánica. El síndrome esencialista nos prepara para percibirnos como miembros típicos de un grupo pero también como parte de un grupo que comparte características importantes y profundas entre sí. Sin embargo, frente a la similitud autoestereotípica o la homogeneidad intragrupo, estos subtipos de similitud resultan menos explicativos.

Para el caso del esencialismo exogrupal, solo una de las variables del proceso de identificación resultó ser significativa para explicar la adhesión a este tipo de creencias. La autocategorización con el propio grupo, explica el surgimiento de una visión esencialista de otro grupo. Esto podría explicarse por la tendencia de las personas a diferenciar, atenuar y/o enfatizar las características propias entre los miembros de un grupo que tiene como consecuencia también una visión más homogénea de los otros grupos. Esto sucedería porque ordenamos el entorno en categorías sociales (Tajfel, 1957), y acentuamos las similitudes entre los participantes de un grupo y por otro lado enfatizamos las diferencias que poseen sujetos que pertenecen a grupos distintos. Por su parte, el compromiso grupal no resultó significativo para el caso del esencialismo exogrupal. Esto podría explicarse porque los componentes emocionales asociados a este aspecto de la identificación, podrían encontrarse asociados únicamente al propio grupo.
Estando implicados en el proceso de identificación con el grupo elementos perceptivos, afectivos y emocionales que la conforman como un proceso actitudinal complejo, los efectos pueden prolongarse al espacio intergrupal, pero sin olvidar que sus consecuencias principales ocurren en lo intragrupal. Asimismo, la autoestima grupal, como componente de la identificación no mostró ser explicativa para el esencialismo exogrupal. Siendo la autoestima un comportamiento asociado a procesos intragrupales, su efecto parece concentrarse solo en este territorio (Castano, 2004). El en caso de la similitud, la percepción de homogeneidad del exogrupo resultó explicativa de las creencias esencialistas sobre el mismo. Esto resulta coherente con la literatura existente que indica que la percepción esencialista se acompaña de la percepción de entitatividad, es decir, se percibe que el grupo posee elementos semejantes entre cada uno de sus miembros, por lo que la entitatividad hará que el grupo parezca una entidad coherente, definida y unitaria (Campbell, 1958). La entitatividad del propio grupo tiene como consecuencia la entitatividad del exogrupo ya que implica fuertes componentes perceptivos que tienen efectos en las relaciones intergrupales (Young, Sánchez, \& Wilton, 2013). Los otros subtipos de similitud parecen tener un impacto específico en el intragrupo y no participan de la percepción de las relaciones intergrupales. El autoestereotipo hace referencia a la percepción que tiene el sujeto sobre las características que debiese tener un representante típico de su grupo, por lo que se hace coherente que resultara no significativo. La similitud intragrupal se establece a partir de la existencia de una "esencia común" y las diferencias entre los grupos se basa justamente en no compartir dicha "esencia" (Estrada et al., 2004). En conclusión, la hipótesis inicial de que los procesos, tanto de identificación con el grupo como el de percepción de similitud, resultan relevantes como variables explicativas del esencialismo. Ambas, comparten algunas características con el fenómeno esencialista que determinan su fuerte asociación. Sin embargo al analizar al esencialismo del propio grupo (endogrupo) y de la percepción esencialista de otro grupo (exogrupo), la identificación y la percepción de similitud no actúan de la misma manera. Los resultados parecen indicar que estas medidas son particularmente importantes en el estudio del esencialismo endogrupal y que tienen algunas consecuencias en la percepción de un exogrupo como resultado de su efecto inicial en el espacio intragrupal. Es así como la identificación en su aspecto emocional (compromiso grupal), que es un fenómeno intragrupal, es relevante para el esencialismo endogrupal pero no extiende su efecto hacia el esencialismo exogrupal. Los aspectos más puramente cognitivos, es decir, que tienen impacto en la percepción de las categorías sociales, se presentan para ambos tipos de esencialismo.

Una de las limitaciones evidentes del presente estudio es que fue realizado con categorías sociales naturales. Resulta indispensable generar nuevas investigaciones con grupos experimentales de forma a poder observar el comportamiento de las variables en una condición más controlada. Es posible que los resultados, por ejemplo, el del compromiso emocional, puedan variar al tratarse de categorías sociales desvinculadas de una identidad social real. Otras variables deberán ser incluidas para profundizar el estudio del 
esencialismo tales como la percepción o no de conflicto entre los grupos y la positividad de las categorías implicadas.

La presente investigación pretende ser un aporte inicial a la comprensión de las variables mediadoras del esencialismo psicológico y de su dinámica como proceso intra e intergrupal.

\section{Referencias}

Alarcón, P., \& Estrada, C. (2011). Estudio de estereotipo y metaestereotipo de una muestra de población chilota residente en la ciudad de Punta Arenas. Salud \& Sociedad, 2(3), 299-309.

Allport, G. (1954). The nature of prejudice. Reading, MA: Addison Wesley.

Bastian, B., \& Haslam. N. (2008). Psychological essentialism and social identification: Immigration from two perspectives. Asian Journal of Social Psychology, 11, 127-140

Brewer, M. B. (1991). The social self - On being the same and different at the same time. Personality and Social Psychology Bulletin. 17(5), 475-482

Byrne, D. (1971). The attraction paradigm. New York: Academic Press.

Calquín, D., \& Estrada, C. (2011). Esencialismo, su impacto en la percepción de similitud y el uso de diferente tipo de información en la formación de impresión. Psykhe, 20(1), 63-75.

Campbell, D. T. (1958). Common fate, similarity, and other indices of the status of aggregates of persons as social entities. Behavioral Science, 3, 14-25

Castano, E. (2004). In case of death, cling to the ingroup. European Journal of Social Psychology, 34(4), 375-384.

Codol, J. P. (1979). Semblables et différents. Recherche sur la quête de la similitude et de la différenciation sociale [Semejantes y diferentes. Investigación sobre la búsqueda de la similitud y la diferenciación social] (Unpublished Doctoral Dissertation). Université de Provence, Aix en Provence, Francia.

Corneille, O., \& Leyens, J-Ph. (1994). Catégories, catégorisation sociale et essentialisme psychologique. In R. Y. Bourhis \& $\mathrm{J}$ - Ph. Leyens (Eds.), Stéréotypes, discrimination et relations intergroupes (pp. 4268). Bruxelles: Mardaga.

Demoulin, S., Leyens, J.-Ph., \& Yzerbyt, V. Y. (2006). Lay theories of essentialism. Group Processes and Intergroup Relations, 9, 25-42.

Ellemers, N., Kortekaas, P., \& Ouwerkerk, J. (1999). Selfcategorization, commitment to the group and group self-esteem as related but distinct aspect of social identity. European Journal of Social Psychology, 29, 371-389.

Estrada, C. (2003). L'impact de l'essentialisme psychologique sur les processus intra- et intergroupe (Unpublished doctoral dissertation). Universidad Católica de Lovaina, Lovaina la Nueva, Bélgica.

Estrada, C. (2009). El impacto del esencialismo psicológico sobre la percepción de la disidencia intragrupal. Interamerican Journal of Psychology, 44(2), 352-360.

Estrada, C., \& Avendaño, S. (2008). ¿Consideran los magallánicos que su cultura posee una esencia única?: Estudio descriptivo de la aplicación de un instrumento de evaluación de esencialismo cultural a una muestra puntarenense. Magallania, 36(2), 155162.
Estrada, C., Oyarzún, M., \& Yzerbyt, V. (2007). Teorías implícitas y esencialismo psicológico: Herramientas conceptuales para el estudio de las relaciones entre y dentro de los grupos. Psykhe, 16(1), 111-121.

Estrada, C., Yzerbyt, V., \& Seron, E. (2004). Efecto del esencialismo psicológico sobre las teorías ingenuas de las diferencias grupales. Psicothema, 16(2), 81-186.

Hinkle, S., Taylor, L., Fox-Cardamone, L., \& Crook, K. (2011). Intragroup identification and intergroup differentiation: A multicomponent approach. British Journal of Social Psychology, 28(4), 305-317.

Kamans, E., Gordijn, E., Oldenhuis, H., \& Otten, S. (2009). What I think, you see is what you get: Influence of prejudice on assimilation to negative meta-stereotypes among Dutch Moroccan teenagers. European Journal of Social Psychology, 39, 842-851.

Leyens, J-Ph., Paladino, P. M., Rodríguez-Torres, R., Vaes, J. Demoulin, S., \& Rodriguez-Pérez, A. (2000). The emotional side of prejudice. The attribution of secondary emotions to ingroups and outgroups. Personality and Social Psychology Review, 4, 186-197.

Leyens, J. Ph., Rodríguez-Pérez, Rodríguez-Torres, R., Gaunt, R., Paladino, M.P., \& Vaes, J. (2001). Psychological essentialism and the differential attribution of uniquely human emotions to ingroups. European Journal of Social Psychology, 31, 395-411.

Martin, C. L., \& Parker, S. (1995). Folk theories about sex and race differences. Personality and Social Psychology Bulletin, 21, 45-57.

Medin, D. L. (1989). Concepts and conceptual structure. American Psychologist, 44, 1469-1481.

Montalbán, F., \& Gómez, L. (1995). Efectos de las discrepancias de estatus sobre el comportamiento intergrupal. Revista Psicología Social, 10(2), 219-233.

Morton, T. A., \& Postmes, T. (2009). When differences become essential: Minority essentialism in response to majority treatment. Personality and Social Psychology Bulletin, 35, 656-668. doi:10.1177/0146167208331254

Pehrson, S., Brown, R., \& Zagefka, H. (2009). When does national identification lead to the rejection of immigrants? Crosssectional and longitudinal evidence for the role of essentialist in-group definitions. British Journal of Social Psychology, 48, 61-76. doi:10.1348/014466608X288827

Pereira, M., Estramiana, J. L., Vasconcelos, C., \& Alves, M. (2010). Essentialistic belief about policemen and criminals. Psicologia: Teoria e Pesquisa, 26(4), 707-715

Pereira, M., Alvaro, J. L., Oliveira, A., \& Dantas, G. (2011). Stereotypes and the essencialization of black and White people: a comparative study. Psicologia \& Sociedade, 23(1), 144 - 153

Rothbart, M. \& Taylor, M. (1992). Category label and social reality: Do we view social categories as natural kinds? In K. Semin \& A. Fiedler (Eds.), Language, interaction and social cognition (pp. 11-36). London: Sage.

Sherif, M. (1948). An outline of social psychology. New York: Harper and Row.

Tajfel, H. (1957). Value and the perceptual judgement of magnitude. Psychological Review, 64, 192-204

Tajfel, H. (1984). Grupos humanos y categorías sociales. Barcelona: Herder. 
Turner, J. C. (1982). Towards a cognitive redefinition of the social group. In H. Tajfel (Ed.), Social identity and intergroup relations (pp. 15-40). Cambridge: Cambridge University Press.

Wagner, W., Holtz, P., \& Kashima, Y. (2009). Construction and deconstruction of essence in representing social groups: Identity projects, stereotyping, and racism. Journal for the Theory of Social Behavior, 39, 363-383.

Young, D. M., Sanchez, D. T., \& Wilton, L. S. (2013). At the Crossroad of Race: Racial ambiguity and biracial identification influence psychological essentialist thinking. Cultural Diversityand Ethnic Minority Psychology. 19(4), 461-467.

Yzerbyt, V., \& Demoulin, S. (2010). Intergroup relations. In S. T. Fiske, D. T. Gilbert \& G. Lindzey (Eds.), The Handbook of Social Psychology (5th edition, Vol 2, pp. 1024-1083). Honoboken NJ: Wiley

Yzerbyt,V., \& Rocher, (2002). Subjective essentialism and the emergence of stereotypes. In C McGarty, V. Yzerbyt \& R. Spears (Eds.), Stereotypes as explanations: The formation of meaningful belief about social groups (pp. 38-66). Cambridge: University Press.
Yzerbyt, V., \& Schadron, G. (1996). Connaitre et juger autrui: Une introduction à la cognition sociale [Conocer y juzgar al otro: una introducción a la cognición social]. Grenoble, Francia: Presses Universitaires de Grenoble.

Yzerbyt, V., Judd, C., \& Corneille, O. (2003). The Psychology of group perception. New York: Psychology Press.

Yzerbyt, V., Rocher, S., \& Schadron, G. (1997). Stereotypes as explanations: A subjective essentialist view of group perception: In R. Spears, P. J. Oakes, N. Ellemers \& S. A. Haslam (Eds.), The Social Psychology of Stereotyping and Group Life. Oxford: Blackwell.

Zagefka, H., Nigbur, D., González, R., \& Tip, L. (2013). Why does ingroup essentialism increase prejudice against minority members? International Journal of Psychology, 48(1), 60-68

Recebido em 27.01.2014

Primeira decisão editorial em 09.11.2014

Versão final em 20.05.2015

Aceito em 08.07.2015 